\title{
The Frascati LINAC beam facility performance and upgrades
}

\author{
Paolo Valente* on behalf of the BTF upgrade team $^{\dagger}$ \\ Istituto Nazionale di Fisica Nucleare, Sezione di Roma \\ P.le A. Moro 2, I-00185 Roma (Italy). \\ E-mail: paolo.valente@romal.infn.it
}

\begin{abstract}
Test beam and irradiation facilities are key enabling infrastructures for research in HEP and astroparticles. In the last 11 years the Beam-Test Facility (BTF) of the DAФNE accelerator complex, in the Frascati Laboratory, has gained an important role in the European infrastructures devoted to the development and testing of particle detectors. Electron or positron beam can be extracted before the injection into the damping ring - to a dedicated transfer line, where a system composed by a target plus a dipole and collimating slits, can attenuate and select the momentum of secondary particles in narrow $(<1 \%)$ band. The secondary beam is then driven to a dedicated experimental hall for beam-test activities.

The facility can provide run-time tuneable electrons and positrons beams in a defined range of different parameters: energy (up to $750 \mathrm{MeV}$ for $e^{-}$and $550 \mathrm{MeV}$ for $e^{+}$), charge ( up to $10^{10}$ particles/bunch) and pulse length (1.4-40 ns). The bunch delivering rate is depending on the DAФNE injections (up to $49 \mathrm{~Hz}$ ). The electron beam spot and divergence can be adjusted, down to sub-mm sizes and approximately $2 \mathrm{mrad}$ in air. Photons can be produced on a Bremsstrahlung active target, and energy-tagged inside a dipole magnet by means of Silicon micro-strip detectors. Overall, an average of 200 beam days is delivered to about 20 experimental groups each year. The possibility of delivering an high-intensity positron beam, opens the opportunity of performing positron annihilation missing mass experiments for the search of light dark matter particles, like dark photons: recently the PADME experiment has been approved by INFN for running at the BTF starting in 2018.

An important upgrade program of the facility is under way, along three main lines: consolidation of the LINAC infrastructure, in order to guarantee a stable operation in the longer term; upgrade of the LINAC energy, in order to increase the facility capability (especially for the almost unique extracted positron beam); doubling of the BTF beam-lines, in order to cope with the significant increase of users due to the much wider range of applications.
\end{abstract}

38th International Conference on High Energy Physics

3-10 August 2016

Chicago, USA

*Speaker.

${ }^{\dagger}$ M. Belli, B. Bolli, B. Buonomo, S. Cantarella, R. Ceccarelli, A. Cecchinelli, O. Cerafogli, R. Clementi, C. Di Giulio, A. Esposito, O. Frasciello, L. G. Foggetta, A. Ghigo, S. Incremona, F. Iungo, R. Mascio, S. Martelli, G. Piermarini, L. Sabbatini, F. Sardone, G. Sensolini, R. Ricci, L. A. Rossi, U. Rotundo, A. Stella, S. Strabioli, R. Zarlenga (INFN Laboratori Nazionali di Frascati); P. Valente (INFN Roma). 


\section{Introduction}

The Beam-Test Facilty (BTF), part of the DAФNE accelerator complex in the Frascati Laboratory, is a beam extraction and manipulation line optimized for the production of "single" electrons. Since its commissioning in 2002 [1], and the installation of a dedicated pulsed extraction magnet in 2004 [2], BTF has continuously improved the beam flexibility, availability and monitoring, at the same time extending the beam parameters range, so that it has gained an important role in the European infrastructures devoted to the development and testing of particle detectors. This has been possible also thanks to the variety of services made available to the users: power supply, networking, gas system, DAQ and beam diagnostics, vacuum and cryogenics, alignment, and magnetic fields [3].

The BTF makes use of electron or positron beam pulses accelerated by the DA $\Phi$ NE LINAC [4] at $50 \mathrm{~Hz}$ maximum repetition rate The DAФNE LINAC is a S-band traveling wave $2 / 3 \pi$, constant gradient, linear accelerator using a high-current thermo-ionic gun, and is composed by 15 SLACtype, $3 \mathrm{~m}$ long, accelerating sections, fed by four RF stations based on $45 \mathrm{MWp}$ klystrons with SLED pulse compression. The first five sections bring electrons emitted by the gun and bunched by the pre-buncher and buncher structures to a maximum energy of $\approx 220 \mathrm{MeV}$, and are followed by a target for positron production; positrons can then be accelerated up to $\approx 550 \mathrm{MeV}$ by the following ten sections. When operating without the positron converter, electrons can be accelerated up to $\approx 750 \mathrm{MeV}$.

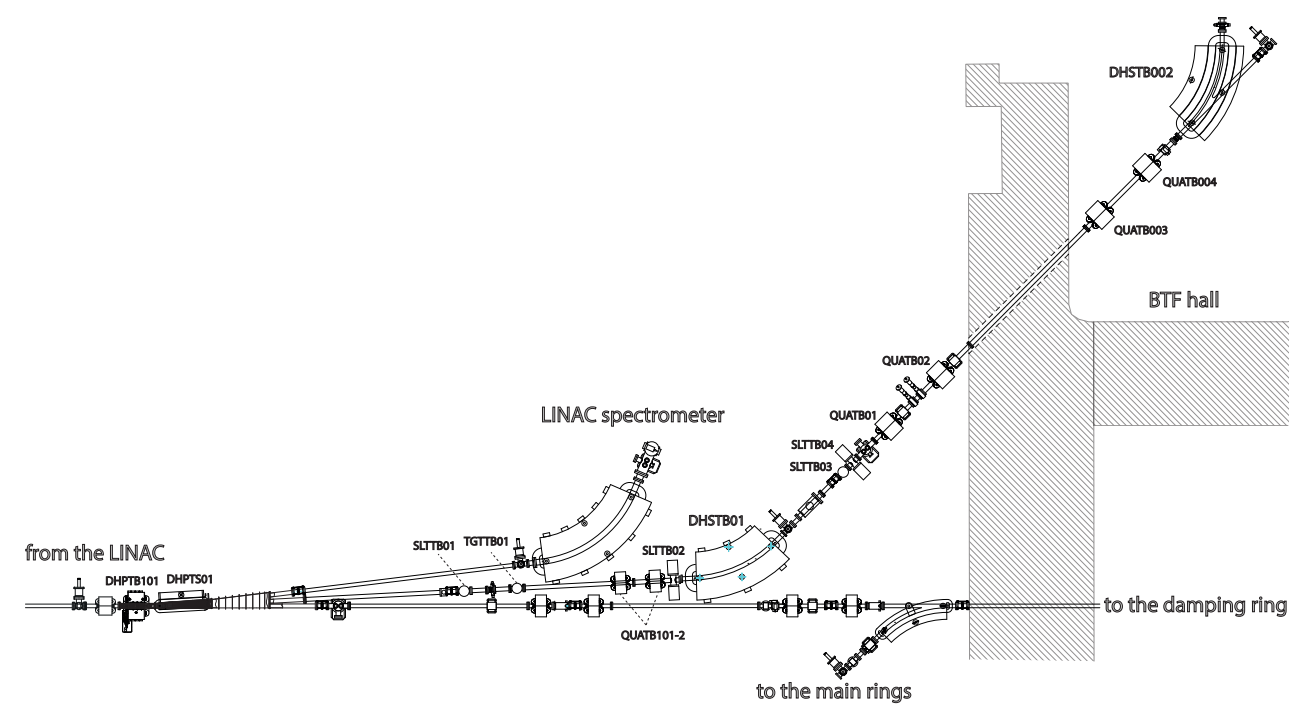

Figure 1: Layout of the existing BTF line.

During the DAФNE collider operation, $510 \mathrm{MeV}$ electron and positron pulses of 1-2 $\mathrm{nC}$ charge and $10 \mathrm{~ns}$ lenght are injected into the damping ring (and from there delivered to the main rings): this is done using the main, straight transfer line shown in Fig. 1). Once per second (twice during injections), a single LINAC pulse is deviated by $6^{\circ}$, by means of the pulsed magnet $\mathrm{DH}-$ PTS01 towards a $60^{\circ}$ spectrometer, for the precise measurement of the beam momentum. All LINAC pulses not used for the injections or directed to the spectrometer line are available for the test-facility, and are sent to the BTF line by means of the $3^{\circ}$ pulsed magnet DHPTB101. 


\section{BTF status}

The BTF has been designed for working in parallel with the operation of the collider, profiting of all LINAC bunches not used for injections, in two main intensity regimes: extracting the full beam of up to $\sim 10^{10}$ particles at the fixed energy of the LINAC (typically $E_{0}=510 \mathrm{MeV}$ ), or by strongly attenuating it by means a Copper target, two radiation lengths thick (TGTTB01, all labels in this section referring to Fig. 1) and re-selecting secondary electrons or positrons in a wide energy range (from few tens of $\mathrm{MeV}$ to $E_{0}$ ), by means of a dipole magnetic selector (DHSTB01) plus collimators. The following transfer line, composed by two quadrupole doublets (QUATB01-02 and QUATB03-04) and a dipole (DHSTB02) allows controlling the beam optics, while collimators and the "capture" doublet (QUATB101-102) immediately after the target allow a fine control of the beam intensity in the full energy range, down to the "single particle" regime (Poisson distribution).

Rich diagnostics allows controlling and monitoring the beam main paramenters: calorimeters, Silicon pixel detectors in the low and intermediate intensity regime, and fluorescent flags and integrating current toroid at high intensity. In Fig. 2 an example of beam spot measurement by means of FitPIX Silicon pixel detector at different beam energies is shown [5].

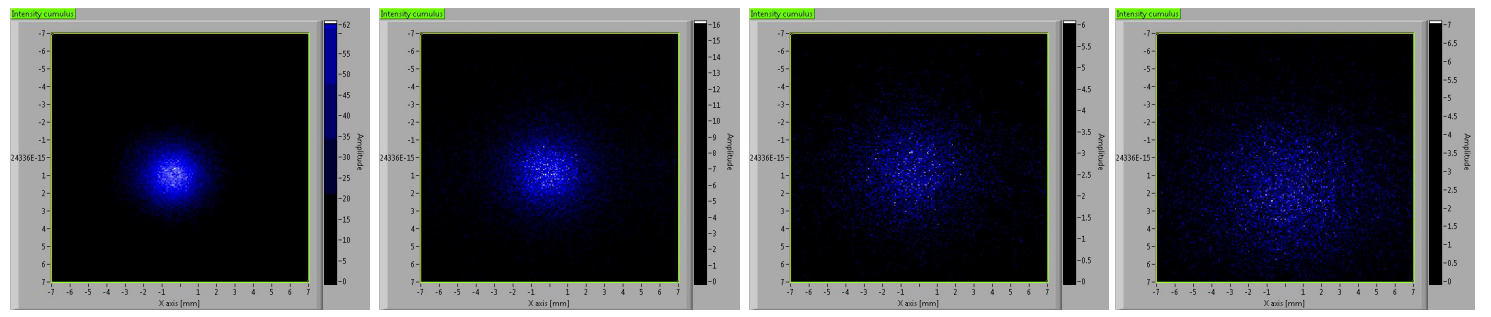

Figure 2: From left to right: BTF beam spots measured by means of a FitPIX Silicon detector ( $55 \mu \mathrm{m}$ pixel side) for $E=150,120,90$ and $60 \mathrm{MeV}$; the dominant contribution to the spot enlargement is the multiple scattering on the $500 \mu \mathrm{m}$ Beryllium beam exit window.

A tagged photons source is also available, constituted by an active target for the production of Bremsstrahlung photons, made by silicon micro-strip detector planes, and making use of a series of modules of silicon micro-strip detectors placed in the last $45^{\circ}$ dipole (DHSTB02) pole gap, for the measurement of the radiating electron energy loss. The system has been designed in collaboration with the AGILE satellite team, with the aim of calibrating the scientific payload before the launch, with a well defined energy and impact point photon beam [7].

\section{BTF upgrades}

An important upgrade program of the facility is under way, along three main lines [8]: consolidation of the LINAC infrastructure, in order to guarantee a stable operation in the longer term; upgrade of the LINAC energy, in order to increase the facility capability (especially for the almost unique extracted positron beam); doubling of the BTF beam-lines, in order to cope with the significant increase of users due to the increasing range of applications.

The first package consists mainly in the consolidation of the LINAC modulators, together with the realization of a complete RF power test-station. These two activities are strongly related, since 
the additional RF station is a fundamental element for the preparation and testing of the components needed for the consolidation of the existing four modulators, reducing to a minimum the LINAC shutdown period.

The simplest scheme for the energy upgrade of the LINAC exploits the extra-length in the LINAC tunnel, of about $15 \mathrm{~m}$, between the last accelerating section at the end of the LINAC and the three-way switch-yard between the damping ring (straight line), and the BTF (at $3^{\circ}$ ) and the spectrometer $\left(\right.$ at $6^{\circ}$ ) lines. This drift space, shown on the bottom left of Fig. 3, presently hosts just four quadrupoles for preserving the beam optics. The additional four sections would require a fifth RF power station, identical to the existing ones, increasing the maximum electron energy from $750 \mathrm{MeV}$ to $\approx 1 \mathrm{GeV}$.

An alternative approach implies increasing the RF power in the existing accelerating section (and in the possible four additional ones), by adding klystrons and SLED's and the relative modulators. Apart from cost considerations, this is a more complex operation from the infrastructural point of view, even though the accelerating gradient would be significantly increased, e.g. from $17 \mathrm{MV} / \mathrm{m}$ to $\approx 24 \mathrm{MV} / \mathrm{m}$ in case the RF power is doubled (feeding two instead that four sections/station).

The doubling of the test lines is strongly motivated by the increasing request for beam in the last years of operation of the facility. Overall, an average of 200 beam days are delivered to about 20 experimental groups each year; in addition, the PADME experiment has been approved by INFN for running at the BTF starting from the beginning of 2018, for searching dark photons and other dark sector light particles in positron annihilations onto the electrons of a target, using the missing mass technique [6]. The idea is to realize a second beam-line, splitting the existing one at the entrance of the present experimental hall and driving a beam branch into the area presently used as control room, as shown in Fig. 3.

The major issue consists in the very limited space available for turning the beam by additional $135^{\circ}$, in order to direct it along the second experimental hall, while preserving the beam quality and, at the same time, keeping the flexibility of energy and intensity tuning. This can be achieved dividing the overall bending required for the new line (labelled BTF-2) among four dipoles: the first of just $15^{\circ}$, dubbed DP01 in Fig. 3, should allow a fast-ramping on and off, in order to achieve a quick and efficient switching among the two lines; the other three, DH01 and DH02 of $45^{\circ}$, and $\mathrm{DC} 01$ of $30^{\circ}$, are followed by quadrupole doublets for keeping control of the beam focussing. Additional beam attenuation and collimation can also be performed by means of a thin (with respect to the main one, TGTTB01) target TGT02, and by the collimator SLT06.

The original line (dubbed BTF-1 in the new layout) would be practically unchanged, also reusing the existing DHSTB02 dipole, with some additional beam control by means of an additional (vertical) corrector and (horizontal) collimator slits SLT05. Simulations of the new transfer line with MAD-X and G4-beamline confirm that the new layout is compliant with the requirement of preserving beam parameters similar to the ones of the present BTF line [9], for both the BTF-1 and BTF-2 branches.

Furthermore, in order to drive a significant particle fluence, a shielding has to be realized for the second experimental hall. The more flexible option is to use removable blocks for realizing a new bunker; preliminary studies show that a dose rate below $3 \cdot 10^{-5} \mathrm{pSv} /$ primary is reached with with $1 \mathrm{~m}$ of concrete. 


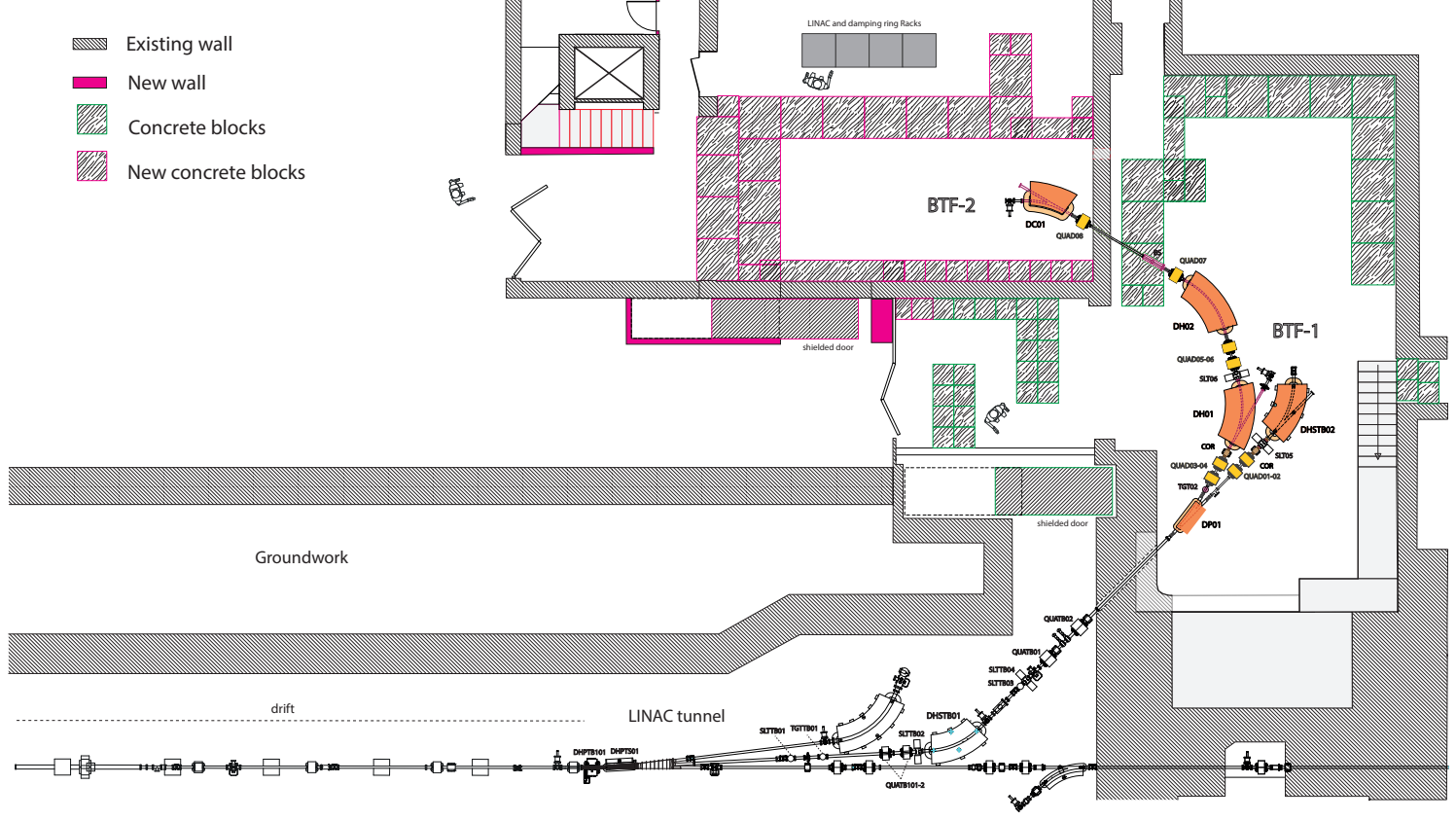

Figure 3: Layout of the new BTF lines.

\section{Conclusions}

In the last 11 years, the DA $\Phi N E B T F$ has provided beam to a increasingly wide community in high-energy and astroparticle physics, with the main focus on the development and testing of particle detectors. The control of the beam parameters, as well as the diagnostics and services available to the users has been constantly improving. In order to keep the present level of availability, it is necessary to extend the access capability, by doubling the existing beam-line, while keeping the flexibility and quality of the beam. At the same time, a strong consolidation program for extending the lifetime of the LINAC is under way.

\section{Acknowledgments}

This work is supported by the H2020 project AIDA-2020, GA no. 654168.

\section{References}

[1] A. Ghigo, G. Mazzitelli, F. Sannibale, P. Valente and G. Vignola, Nucl. Instrum. Meth. A 515, 524 (2003).

[2] P. Valente, B. Buonomo and G. Mazzitelli, Nucl. Phys. Proc. Suppl. 150, 362 (2006).

[3] L. G. Foggetta, B. Buonomo and P. Valente, C15-05-03, p. MOPHA049 (2015).

[4] B. Buonomo and L. G. Foggetta, C-15-05-03, p. TUPWA057 (2015). 
[5] L. G. Foggetta, B. Buonomo and P. Valente, C15-05-03, p. MOPHA048 (2015).

[6] P. Valente et al., "The Frascati LINAC beam facility performance and upgrades", these Proceedings.

[7] P. W. Cattaneo et al., Nucl. Instrum. Meth. A 674, 55 (2012).

[8] P. Valente et al., arXiv:1603.05651 [physics.acc-ph].

[9] B. Buonomo et al., AIDA-2020-NOTE-2016-001. 